\title{
Estudio comparativo in vitro del grado de penetración y microfiltración de dos selladores de fosas y fisuras (ionomérico y resinas): influencia en la técnica de aplicación
}

\author{
In vitro comparative study of penetration and degree of microfiltration of two pits and fissures \\ sealants (ionomeric and composite resins): influence on the application technique
}

\author{
Jéssica Avilés Erazo ${ }^{1 a}$, Ana Armas Vega ${ }^{1 b}$, Paola Mena Silva 1,2b, Sebastián Chiluisa Cueva ${ }^{2 c}$, Viviana Hidalgo Moya ${ }^{1 a}$, \\ Isadora Martini García ${ }^{3 b}$, Fabricio Mezzomo Collares ${ }^{3 b}$, Edison López Ríos ${ }^{1 b}$
}

\section{RESUMEN}

Objetivos: comparar la capacidad de penetración y el grado de microfiltración entre un sellador resinoso y un ionomérico, con y sin ameloplastía; y, con o sin la colocación de un adhesivo previo a la colocación del sellante resinoso. Metodología: La muestra estuvo compuesta por 90 premolares libres de caries, con posterior profilaxis. Fueron divididos en seis grupos, G1: se realizó ameloplastía, grabado ácido y aplicación de sellante resinoso, G2: ameloplastía, grabado ácido, aplicación de adhesivo y sellante resinoso, G3: grabado ácido, aplicación de adhesivo y sellante resinoso, G4: aplicación de sellante ionomérico, G5: grabado ácido y aplicación de sellante resinoso, G6: ameloplastía y aplicación de sellante ionomérico. Tras el termociclado, los dientes fueron seccionados y observados los fragmentos bajo estereomicroscopio y observación al Microscopio Electrónico de Barrido. Resultados: el menor grado de microfiltración fue detectado en el grupo sometido a ameloplastía, grabado ácido, aplicación de adhesivo y sellante resinoso $(0,198 \mathrm{~mm})$. Existió mayor penetración del sellante con la técnica de ameloplastía. Conclusiones: La colocación de adhesivo previo al sellante resinoso influyó en el grado de microfiltración y la realización de ameloplastía ayuda con una mayor penetración del material sellador.

PALABRAS CLAVES: Sellantes, Ionómero, Diente, Microfiltración. (Fuente: DeCS BIREME)

\begin{abstract}
:
Objetives: to compare the penetration capacity and the degree of microfiltration between a resinous sealant and an ionomer with and without ameloplasty, and with or without the placement of an adhesive prior to the placement of the resinous sealant. Methodology: 90 donated premolars, free of caries obtained previous indication orthodontic, later prophylaxis, were divided into six groups, in G1: ameloplasty was performed, acid etching, resinous sealant application, G2: ameloplasty, acid etching, adhesive application and sealant resinous, G3: acid etching, application of adhesive and resinous sealant, G4: application of ionomeric sealant, G5: acid etching, application of resinous sealant, G6: ameloplasty, application of ionomeric sealant. After thermocycling, the teeth were sectioned and the fragments observed under stereomicroscope and observation to the Scanning Electron Microscope. Results: he lowest degree of microfiltration was detected in the group undergoing ameloplasty, acid etching, application of adhesive and resinous sealant $(0.198 \mathrm{~mm})$. There was greater penetration of the sealant with the ameloplasty technique. Conclusions: The placement of adhesive prior to the resinous sealant influenced the degree of microfiltration, and the performance of ameloplasty helps with greater penetration of the sealant material.
\end{abstract}

KEY WORDS: Sealants; Ionomer; Tooth; Microfiltration. (Source: MeSH NLM)

\footnotetext{
${ }^{1}$ Universidad Central Del Ecuador, Facultad de Odontología, Quito, Ecuador.

2 Universidad Regional Autónoma de los Andes, Facultad de Odontología,

Ambato, Ecuador

${ }^{3}$ Universidad Federal Rio Grande del Sur, Facultad de Odontología. Porto Alegre, Brasil.

a Odontóloga Genera

${ }^{b} \mathrm{PhD}$. En Odontología

${ }^{b}$ Estudiante Carrera de Odontología
}

Este es un artículo Open Access distribuido bajo la licencia Creative Commons Atribución-No Comercial-

Compartir Igual 4.0

Correspondencia:

Juan Pablo Rodríguez Villarreal

Humberto Albornoz y Barros de San Millan OE 6 - 155. Quito, Ecuador.

Celular: +593 982036238 Correo electrónico: pablorodri-@hotmail.es

Citar como: Aviles J, Armas A, Mena P, Chiluisa S, Hidalgo V, Martini I, Mezzomo F, López E. Estudio comparativo in vitro del grado de penetración y microfiltración de dos selladores de fosas y fisuras (ionomérico y resinas): influencia en la técnica de aplicación KIRU. 2020; 17(1): 62- 68. https://doi.org/10.24265/kiru.2020.v17n2.01 


\section{INTRODUCCIÓN}

La odontología ha evolucionado a lo largo de la historia. Anteriormente los recursos existentes eran limitados, lo que imposibilitaba instaurar alternativas conservadoras y preventivas, dando lugar a la pérdida de dientes; con el devenir de los años, la terapéutica preventiva marca un efecto positivo en la integridad dental con la disminución de las patologías dentales ${ }^{1,2}$.

Se ha observado una mayor susceptibilidad de la caries en fosas y fisuras, con una mayor prevalencia entre el $50 \%$ y $95 \%{ }^{3}$, pero existe una alta probabilidad de interceptar esta patología de manera temprana ${ }^{4,5}$. Los sellantes constituyen un método preventivo que forma una barrera física que impide la proliferación bacteriana en la superficie dental evitando los procesos cariosos ${ }^{6}$.

Los primeros materiales utilizados para sellar fosas y fisuras fueron los poliuretanos, que se descartaron luego por el poco éxito alcanzado, seguido de los cianocrilatos que se desintegraban en poco tiempo ${ }^{3}$, la utilización del grabado ácido al $50 \%$ sobre la superficie dental fue propuesto con la finalidad de obtener mejoras en la adhesión de los materiales utilizados como selladores.

Dentro del campo de la prevención, otro material utilizado con eficacia como sellante de fosas y fisuras es el ionómero vítreo ${ }^{3}$. Muestra como principal característica su adhesión química al esmalte, dentina y cemento $^{7,} 8$; sin embargo, una alternativa para mejorar los procesos retentivos de estos materiales se encuentra en la ameloplastía u odontotomía profiláctica que se plantea como la eliminación quirúrgica de la fisura por medio de instrumental rotatorio ${ }^{6}$. La limpieza de las fosas y fisuras con cepillo profiláctico y pasta abrasiva, para una mejor adaptación de los materiales de sellado ${ }^{9}$, ayudó en la reducción de bacterias debajo del sellante y a una mejor retención al realizar ameloplastía en fosas y fisuras. Una de las variables es la colocación de adhesivo con el fin de lograr una mejor adhesión y una menor microfiltración ${ }^{10}$.

El objetivo de este estudio es comparar, mediante Microscopia Electrónica de Barrido y Estereomicroscopia, la capacidad de penetración y el grado de microfiltración de un sellador de fosas y fisuras resinoso, y un ionomérico, en relación a la técnica de aplicación (ameloplastía y no ameloplastía); y, determinar si el uso de adhesivo, previa la colocación de sellante resinoso, disminuye la microfiltración mejorando la técnica.

La caries dental es una lesión patológica que afecta al tejido dentario mineralizado, Fejerskov et al. (2008) citado por (Henostroza, 2010) mencionó que existe una alteración del equilibrio fisiológico entre los minerales dentarios y los fluidos existentes en el biofilm dental. Nuevas técnicas preventivas se han desarrollado día a día buscando evitar estas lesiones, así nace el presente trabajo de investigación, que espera contribuir con resultados favorables en el manejo de los selladores de fosas y fisuras.

\section{MATERIALES Y MÉTODOS}

Se obtuvo la aprobación del comité de ética de la Universidad Central del Ecuador y se procedió a realizar el estudio comparativo experimental in vitro, se utilizaron 90 premolares extraídos por indicaciones ortodóncicas, sanos, sin presencia de material alguno en la superficie, con surcos y fosas marcadas, la muestra obtenida fue dividida al azar en 6 grupos de 15 piezas.

Preparación de las muestras: La superficie oclusal de los 90 premolares fueron limpiadas con pasta de piedra pómez y agua destilada con cepillo profiláctico y micromotor. Se utilizó la punta de un explorador para eliminar cualquier residuo existente. En el primer grupo (G1), en cada diente se realizó ameloplastía en las fisuras con una fresa redonda $1 / 4$ de carburo tungsteno (Jet, Ontario, Canadá), se procedió a lavar con agua por 15 segundos, secando posteriormente cada superficie, se aplicó ácido fosfórico al $35 \%$ (3M-Espe) durante 15 segundos mediante la jeringa aplicadora, lavado posterior durante 15 segundos, posterior secado con la jeringa triple, aplicando a continuación lentamente el sellante resinoso (Clinpro 3M-ESPE) dispensando con la jeringa con boquilla aplicadora y verificando que no existan burbujas y fotopolimerizando durante 20 segundos con lámpara de luz halógena (Litex 680 A) a $1 \mathrm{~cm}$ aproximadamente de la pieza dental. (Figura 1A)

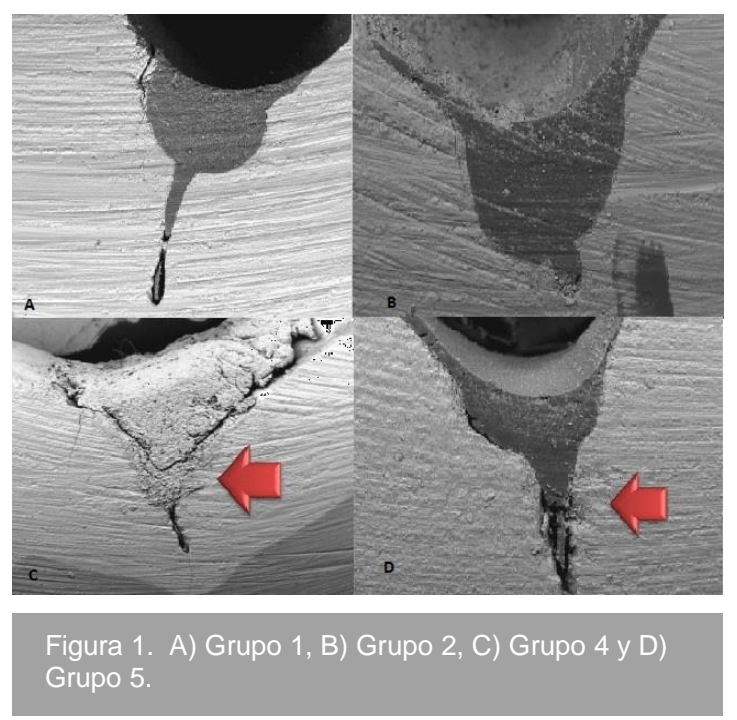

En el segundo grupo (G2), en cada diente se realizó ameloplastía en las fisuras con una fresa redonda $1 / 4$ de carburo tungsteno (Jet, Ontario, Canadá), lavado 
con agua por 15 segundos, secando posteriormente cada diente, recibieron aplicación de ácido fosfórico al $35 \%$ (3M-Espe), durante 15 segundos mediante la jeringa aplicadora, lavado posterior durante 15 segundos con agua, secado suavemente con la jeringa triple a $1 \mathrm{~cm}$ de distancia, aplicación de adhesivo (Single bond 3M-ESPE) suministrando a continuación lentamente el sellante resinoso (Clinpro 3M-ESPE) y verificando que no existan burbujas y fotopolimerizando durante 20 segundos. (Figura 1B) En el tercer grupo (G3), cada diente recibió aplicación de ácido fosfórico al $35 \%$, (3MEspe), durante 15 segundos mediante la jeringa aplicadora, lavado posterior durante 15 segundos con agua, secado levemente con la jeringa triple a $1 \mathrm{~cm}$ de distancia, aplicación de adhesivo (Single bond 3MESPE) tal como lo descrito en el G1.

En el cuarto grupo (G4), se procedió a la colocación de ácido poliacrílico durante 10 segundos con una bolita de algodón, se lavó con agua de la jeringa triple, secando suavemente con aire, dosificación en el block de papel 1 cucharada rasa de polvo (3,6 gr) y 1 gota dispensada en forma vertical del líquido (1,0 gr) del sellante ionomérico (Fuji IX), con una espátula se dividió el polvo en 2 partes iguales, mezclando la primera parte con todo el líquido durante 10 segundos, seguidamente se incorpora la parte restante y se mezcla durante 15 segundos más, obteniéndose una mezcla homogénea. Durante un tiempo de trabajo desde el inicio de la mezcla de 2 minutos, se coloca la mezcla en las fisuras utilizando un instrumental de punta fina y verificando que no existan burbujas, se espera su completo fraguado y se aplica por último una capa delgada de vaselina. (Figura $1 \mathrm{C}$ )

En el quinto grupo (G5), cada diente recibió aplicación de ácido fosfórico al 35 \%, (3MEspe), durante 15 segundos mediante la jeringa dispensadora, lavado posterior durante 15 segundos con agua, secado suavemente con la jeringa triple a $1 \mathrm{~cm}$ de distancia, se aplicó a continuación el sellante resinoso (Clinpro 3M-ESPE) tal como lo descrito en el G1. (Figura 1D)

En las muestras del sexto grupo (G6,) se realizó ameloplastía en las fisuras con una fresa redonda $1 / 4$ de carburo tungsteno (Jet, Ontario, Canadá) se lavó a $1 \mathrm{~cm}$ de distancia con agua 15 segundos, secando cada diente de prueba a $1 \mathrm{~cm}$ de distancia con la jeringa triple, seguido de la colocación de ácido poliacrílico durante 10 segundos con una bolita de algodón, lavado con agua durante 15 segundos, se seca suavemente con aire de la jeringa triple, seguido de la dosificación y colocación del sellante ionomérico (Fuji IX) tal cual lo descrito en el G4.

Todos los dientes fueron termociclados en baños de agua caliente y fría, a temperaturas entre 5 a $55^{\circ} \mathrm{C}$ durante 5[00 ciclos, siguiendo el estándar ISO TR 11450 (1994). De cada grupo de estudio se escogieron dos especímenes al azar, quedando así 13 dientes por grupo.
Los dientes seleccionados fueron fragmentados, con disco de diamante (KENDO) en pieza de mano de baja velocidad (NSK). Tres cortes longitudinales a nivel de las fisuras oclusales, en sentido vestíbulolingual 0 vestíbulo-palatino con un espesor aproximado de $1 \mathrm{~mm}$ por cada corte. Posteriormente, se realizó un corte a nivel coronal para desprender los segmentos previamente cortados. Para que no exista variante alguna, se seleccionó la parte media de los tres segmentos seccionados. Los segmentos fueron analizados al Microscopio Electrónico de Barrido (FEl CompanyModelo Quanta 4000), a un voltaje de aceleración de $25.00 \mathrm{KV}$ a $154 \mathrm{X}$ y $1200 \mathrm{X}$, a una distancia de trabajo entre la platina que sostiene la porta muestra y el cañón de electrones de $10 \mathrm{~mm}$ aproximadamente, las microfotografías obtenidas fueron analizadas posteriormente por un investigador con experiencia en la lectura de imágenes.

Análisis para microfiltración: En los dientes restantes de cada grupo, fueron sellados el ápice con resina (P60-3M ESPE) y barniz de uñas, dejando libre solo la superficie que contenía el material sellador aproximadamente a $1 \mathrm{~mm}$; a continuación, se sumergieron las muestras en azul de metileno al $5 \%$ durante 24 horas para su tinción, tras su seccionamiento los dos fragmentos obtenidos fueron observados en el estere microscopio a un rango zoom 0.6X y un aumento de $4 \mathrm{X}$ a $480 \mathrm{X}$ establecido mediante escalímetro para mantener una referencia de medida para la observación del grado de microfiltración. Las mediciones fueron realizadas por dos estudiantes de odontología ajenos al estudio, debidamente entrenados en la ejecución de este procedimiento y en el registro de las mediciones realizadas. De forma complementaria, se obtuvieron imágenes de las muestras mediante la utilización de una cámara fotográfica (NOKIA N82-7MPX). Se procedió a valorar su medida de manera más precisa con la ayuda de Microsoft Publisher utilizando el medidor del mismo, para lo cual de manera referencial se comparó a la fisura junto con el material sellador como un triángulo invertido donde uno de los vértices se dirige hacia apical y los otros dos hacia coronal tomando como punto de inicio uno de los vértices coronales, el más bajo e idóneo, hasta el vértice dirigido hacia apical arrojando así un valor referente. Posteriormente, se dividió en tercios al esquema triangular y se procedió a medir desde el punto de inicio ya establecido a la penetración final del material, así como a la microfiltración del tinte; estas mediciones fueron realizadas por un evaluador entrenado en su ejecución que desconocía los procedimientos y técnicas ejecutadas.

Los resultados obtenidos fueron sometidos a análisis estadístico del programa SPSS versión $X X I$, complementados con las pruebas de ANOVA y Scheffe. 


\section{RESULTADOS}

Los datos obtenidos se compilaron en una hoja de cálculo en el programa Excel, en la que se calculó la relación entre la medida de la microfiltración, penetración y el tamaño de la fisura, se consideró la medida bajo el microscopio obtenida en el análisis de la microfotografía de cada diente. Con estos resultados numéricos, se procedió a diseñar una base de datos en el programa SPSS versión XXI, teniendo en cuenta la relación de microfiltración con el tipo de material empleado y técnica utilizada.
Se observa que la relación de microfiltración versus el tipo de material empleado y técnica utilizada tienen una tendencia similar para 5 de los 6 grupos ensayados, solo el grupo signado como 2; en el que se empleó resina + ameloplastía + adhesivo tuvo un menor grado de microfiltración. Con estos resultados, se procedió a evaluar la prueba de ANOVA (Tabla 1).

Tabla 1. Media del grado de microfiltración según la técnica y el material utilizado.

\begin{tabular}{|c|c|c|c|c|}
\hline Grupo & & Total fisura & Microfiltración & $\begin{array}{l}\text { Microfiltración/ } \\
\text { material }\end{array}$ \\
\hline $\begin{array}{l}\text { Resina } \\
\text { Ameloplastía }\end{array}$ & + & 1,343 & 0,420 & 0,381 \\
\hline \multicolumn{5}{|l|}{ Sin adhesivo } \\
\hline $\begin{array}{l}\text { Resina } \\
\text { Ameloplastía }\end{array}$ & + & 1,233 & 0,198 & 0,190 \\
\hline \multicolumn{5}{|l|}{ + Adhesivo } \\
\hline $\begin{array}{l}\text { Resina } \\
\text { Ameloplastía }\end{array}$ & Sin & 1,292 & 0,272 & 0,280 \\
\hline \multicolumn{5}{|l|}{ + Adhesivo } \\
\hline $\begin{array}{l}\text { Ionómero } \\
\text { Ameloplastía }\end{array}$ & $\operatorname{Sin}$ & 1,308 & 0,308 & 0,368 \\
\hline $\begin{array}{l}\text { Resina } \\
\text { Ameloplastía }\end{array}$ & Sin & 1,504 & 0,345 & 0,357 \\
\hline \multicolumn{5}{|l|}{ Sin adhesivo } \\
\hline $\begin{array}{l}\text { Ionómero } \\
\text { Ameloplastía }\end{array}$ & + & 1,870 & 0,477 & 0,320 \\
\hline Total & & 1,402 & 0,331 & 0,327 \\
\hline
\end{tabular}

La comparación intra e intergrupos nos permitió observar que tanto la microfiltración absoluta como la relación microfiltración versus el material empleado difieren significativamente para los seis grupos de estudio, dado que $p<0,05$ (Tabla 2).

Tabla 2. Resultados de prueba ANOVA inter-intragrupos.

\begin{tabular}{|c|c|c|c|c|c|c|}
\hline & & $\begin{array}{l}\text { Suma } \\
\text { cuadrados }\end{array}$ & gl & $\begin{array}{l}\text { Media } \\
\text { cuadrática }\end{array}$ & $F$ & $p$ \\
\hline \multirow[t]{3}{*}{ Total de Material } & Intergrupos & 3,508 & 5 & ,702 & 11,069 & ,000 \\
\hline & Intragrupos & 4,500 & 71 & ,063 & & \\
\hline & Total & 8,008 & 76 & & & \\
\hline \multirow[t]{3}{*}{ Microfiltración } & Intergrupos & ,682 & 5 & ,136 & 8,517 &, 000 \\
\hline & Intragrupos & 1,137 & 71 & ,016 & & \\
\hline & Total & 1,1819 & 76 & & & \\
\hline \multirow[t]{3}{*}{ Relación mm } & Intergrupos & ,320 & 5 & ,064 & 4,357 & ,002 \\
\hline & Intragrupos & 1,042 & 71 & ,015 & & \\
\hline & Total & 1,362 & 76 & & & \\
\hline
\end{tabular}


La comparación por pares, mediante la prueba de Scheffe, permite inferir que el empleo de adhesivo explica las diferencias significativas en el grado de microfiltración, el uso o no de ameloplastía no es altamente incidental y el uso de resina o ionómero tampoco explican las diferencias halladas en el grado de microfiltración (Tabla 3).

Tabla 3. Resultados de la prueba de Post hoc de Scheffe.

\begin{tabular}{|c|c|c|c|}
\hline Grupo I & Grupo J & $\begin{array}{l}\text { Diferencia de } \\
\text { medias (I-J) }\end{array}$ & Sig. \\
\hline \multirow[t]{5}{*}{ RESINA + AMELOPLASTÍA SB } & $\begin{array}{l}\text { RESINA + AMELOPLASTÍA + } \\
\text { ADHESIVO }\end{array}$ &, $19077^{*}$ & ,011 \\
\hline & $\begin{array}{l}\text { RESINA SIN AMELOPLASTÍA + } \\
\text { ADHESIVO }\end{array}$ & ,03538 & ,990 \\
\hline & $\begin{array}{ll}\text { IONÓMERO } & \text { SIN } \\
\text { AMELOPLASTÍA } & \end{array}$ & ,01308 & 1,000 \\
\hline & $\begin{array}{l}\text { RESINA SIN AMELOPLASTÍA } \\
\text { SIN ADHESIVO }\end{array}$ & 02385 & ,998 \\
\hline & IONÓMERO + AMELOPLASTÍA & ,06077 & ,903 \\
\hline \multirow[t]{5}{*}{$\begin{array}{l}\text { RESINA + AMELOPLASTÍA + } \\
\text { ADHESIVO }\end{array}$} & $\begin{array}{l}\text { RESINA + AMELOPLASTÍA SIN } \\
\text { ADHESIVO }\end{array}$ &,$- 19077^{*}$ & ,011 \\
\hline & $\begin{array}{l}\text { RESINA SIN AMELOPLASTÍA + } \\
\text { ADHESIVO }\end{array}$ &,- 15538 & 071 \\
\hline & $\begin{array}{ll}\text { IONÓMERO } & \text { SIN } \\
\text { AMELOPLASTÍA } & \\
\end{array}$ &,$- 17769^{*}$ & ,023 \\
\hline & $\begin{array}{l}\text { RESINA SIN AMELOPLASTÍA } \\
\text { SIN ADHESIVO }\end{array}$ &,$- 16692^{*}$ & ,041 \\
\hline & IONÓMERO + AMELOPLASTÍA &,- 13000 & ,222 \\
\hline \multirow[t]{5}{*}{$\begin{array}{l}\text { RESINA SIN AMELOPLASTÍA + } \\
\text { ADHESIVO }\end{array}$} & $\begin{array}{l}\text { RESINA + AMELOPLASTÍA SIN } \\
\text { ADHESIVO }\end{array}$ &,- 03538 & ,990 \\
\hline & $\begin{array}{l}\text { RESINA + AMELOPLASTÍA + } \\
\text { ADHESIVO }\end{array}$ & ,15538 & ,071 \\
\hline & $\begin{array}{l}\text { IONÓMERO } \\
\text { AMELOPLASTÍA }\end{array}$ &,- 02231 & ,999 \\
\hline & $\begin{array}{l}\text { RESINA SIN AMELOPLASTÍA } \\
\text { SIN ADHESIVO }\end{array}$ &,- 01154 & 1,000 \\
\hline & IONÓMERO + AMELOPLASTÍA & 02538 & ,998 \\
\hline
\end{tabular}

Se observa que la relación de penetración frente al tamaño de la fisura, no tiene una tendencia clara para los 6 grupos ensayados, se precisó determinar si las diferencias numéricas de las medias del grado de penetración son significativas, para lo cual se desarrolló la prueba de ANOVA. Se observa que tanto la penetración absoluta como la relación penetración versus el tamaño de la fisura difieren para los seis grupos de estudio, dado que $p<0,05$ (Tabla 4).

Tabla 4. Media del grado de Penetración.

\begin{tabular}{|l|l|l|l|}
\hline $\begin{array}{l}\text { GRUPO } \\
\text { RESINA + AMELOPLASTÍA SIN } \\
\text { ADHESIVO }\end{array}$ & 1,343 & 1,085 & 0,828 \\
\hline $\begin{array}{l}\text { RESINA + AMELOPLASTÍA + ADHESIVO } \\
\text { RESINA SIN AMELOPLASTÍA + } \\
\begin{array}{l}\text { ADHESIVO } \\
\text { IONÓMERO SIN AMELOPLASTÍA }\end{array}\end{array}$ & 1,233 & 0,982 & 0,801 \\
\hline $\begin{array}{l}\text { RESINA SIN AMELOPLASTÍA SIN } \\
\text { ADHESIVO }\end{array}$ & 1,504 & 0,789 & 0,678 \\
\hline $\begin{array}{l}\text { IONÓMERO + AMELOPLASTÍA } \\
\text { Total }\end{array}$ & 1,870 & 0,881 & 0,677 \\
\hline
\end{tabular}


La comparación por pares, mediante la prueba de Scheffe, permite inferir que el empleo de ameloplastía podría explicar las diferencias en el grado de penetración, el uso de adhesivo o la diferencia de emplear resina o ionómero no son altamente incidentales.

\section{DISCUSIÓN}

La terapéutica a lo largo del tiempo ha sufrido grandes cambios, los tratamientos hoy en día buscan ser lo más conservadores posibles con el fin de cuidar estructuras que son importantes para el equilibrio de la salud bucal y en general. Al evidenciar que existe una prevalencia de caries dental, se intenta prevenir la aparición de estas lesiones con la utilización de materiales que sellen estas zonas evitando la colonización bacteriana, los cuales llegan a ser un arma importante dentro de nuestro arsenal terapéutico, utilizados con las técnicas y materiales apropiados serán de gran ayuda para la prevención de procesos cariosos ${ }^{7}$.

El mercado odontológico globalizado pone a disposición varios materiales que ayudan a sellar las fosas y fisuras, pero que por ciertas características y variaciones propias en sus fórmulas presentan distintas prestaciones; pretendemos mediante este estudio establecer cuál es el material idóneo para la práctica odontológica, así como, la técnica a emplear.

En nuestro estudio, el uso del escalímetro y el medidor de Publisher con las imágenes obtenidas en el estereomicroscopio nos brindan un resultado objetivo. El escalímetro empleado en diversos estudios científicos ya realizados es reportado como un medio de validez por la evidencia de los resultados brindados; siendo superior al empleo del Microscopio Electrónico de Barrido (MEB); en vista que en este estudio es utilizado como un método subjetivo de evaluación, ya que los resultados quedan a criterio de los evaluadores, el estado de ánimo de los mismos, la predisposición e incluso la experiencia harán que difieran las consideraciones para cada microfotografía, pese a estos reparos el MEB será utilizado como un medio de apoyo y comprobación para la penetración de los materiales selladores, lo que ya fue determinado por las imágenes obtenidas en el estereomicroscopio.

Los datos que se presentan en este estudio, en relación a la penetración del material sellador con la utilización de ameloplastía, dieron resultados significativos: existencia de una mayor penetración de los sellantes de fosas y fisuras en contraste con aquellos en donde no se realizó ameloplastía; sin embargo, a una mejor observación en el Microscopio Electrónico de Barrido (MEB) no existió un sellado completo de las fisuras, quedando así espacios a nivel apical de la fisura, lo que concuerda con las investigaciones previas ${ }^{11,12}$.

Si bien la técnica de ameloplastía mejora la adaptación del sellador al esmalte ${ }^{13}$, resaltando mayor penetración del material sellador, existirá una mejor adaptación por la eliminación de restos residuales, y el incremento del área de superficie ${ }^{4}$, en contraste, este procedimiento atenta los conceptos de máxima conservación del tejido, lo que merece ser analizado minuciosamente en costo beneficio. La colocación del adhesivo no influyó de manera alguna en el índice de penetración como fue evidenciado en las imágenes del estereomicroscopio y en los resultados estadísticos, lo que se explicaría por lo aseverado por Marks ${ }^{14}$, para quien la eficacia del sellante se basa en la unión suficiente en la porción coronal de la fisura sin dependencia de la penetración completa del material en las profundidades de las fisuras ${ }^{15}$, los sellantes en fisuras angostas no penetrarán por completo en la parte inferior de la fisura, por lo que no es necesario la ameloplastía, no hay diferencias en cuanto a eficacia en los selladores previa o no ameloplastía ${ }^{16}$.

Los datos que se presentan en este estudio en relación a un mayor grado de penetración, son auxiliados por la técnica de ameloplastía tanto en sellante resinoso como ionomérico, a diferencia de estudios realizados por Markovic, se observa que los compuestos a base del Bis-GMA presentan una mejor penetración, así como retención en relación a los materiales selladores ionoméricos ${ }^{8}$. Además, al utilizar adhesivo previo, la colocación del material sellador resinoso aumenta la capacidad de penetración del material influyendo además en su retención ${ }^{7}$; sin embargo, se comprobó en el MEB que no existe una penetración completa del material sellador en las fisuras independientemente del material o técnica a utilizarse.

Si bien en la comparación intra e intergrupos se pudo observar diferencias significativas en el grado de microfiltración al emplear adhesivo previo a la colocación del material sellador, con estudios similares se encontró una reducción en el grado de microfiltración explicado por la menor viscosidad del adhesivo y su adherencia al esmalte ${ }^{10-13}$, a diferencia de otros estudios, el uso o no de adhesivos no influyen en el nivel de microfiltración ${ }^{18}$, así como, con la ejecución de ameloplastía o no se observó una mayor microfiltración con materiales ionoméricos ${ }^{11,19}$, coincidiendo con estudios de Alave, G. (2014); Utreras, V. (2016) \& Rojas (2018). 


\section{CONCLUSIONES}

Se pudo observar que la realización de ameloplastía influye en el mayor grado de penetración del material sellador. Además, se pudo diferenciar mediante las fotografías obtenidas en el MEB que no existe una penetración completa del material sellador en las fisuras independientemente del material o técnica a utilizarse.

Los resultados obtenidos demostraron que existe menor microfiltración al realizar ameloplastía, utilización de adhesivo y material sellador resinoso, esto se debe a la influencia del adhesivo, puesto que mediante los estudios de Anova y Scheffe se observó una íntima relación del material sellador con el esmalte.

Pese a las limitaciones del presente estudio por el hecho de ser in vitro y por haber considerado como evaluadores a estudiantes de pregrado de la Facultad de Odontología, quienes posiblemente por su grado de conocimiento o experiencia pudieron actuar como sesgo en el estudio, los resultados de este estudio merecen ser considerados por las implicaciones clínicas que conlleva.

Contribuciones de autoría: DSM, JPRV, AA, EV, VBB y MF diseñaron el estudio, recopilaron y analizaron los datos, redactaron y aprobaron el manuscrito.

Fuente de financiamiento: Autofinanciado.

Conflicto de intereses: Los autores declararon no tener conflictos de interés.

\section{REFERENCIAS}

1. Anusavice, J. (2004). Phillips. Ciencia de los materiales dentales. Madrid-España: El Sevier.

2. Baratieri, L. Odontología Restauradora. Fundamentos y Técnicas. Volumen 1. Editorial Livraria Santos.2011.

3. Barbería, L. (2005). Atlas de Odontología Infantil para Pediatras y Odontólogos. España: Ripano.

4. Barrancos, J. (2002). Operatoria Dental. Buenos Aires: Panamericana.

5. Barrancos, J., Barrancos, P. (2006). Operatoria Dental Integración Clínica. Buenos Aires: Panamericana.

6. Baum, L., Phillips, R., Lund, M. (1984). Tratado de Operatoria Dental. México: Interamericana.

7. Bezerra, L., Borsatto, M., Assed, S. (2008). Sellantes de fosas y fisuras. Caracas, Venezuela: Editorial actualidades médicas odontológicas Latinoamérica. 483-507.
8. Bordoni. N. (2010). Odontología Pediátrica. Editorial Médica Panamericana. Buenos AiresArgentina.

9. Bosan (1993). Mechanical preparation and filling level. J Dent. 28:407-12.

10. Brocklehurst, PR., Joshi, IR., Northeast, S. (1992). the effect or air-polishing occlusal surfaces on the penetration of fissures by a sealant. Int $\mathrm{J}$ Pediatric Dent .2: 157-162

11. Carlsen, O. (1988). Morfología Dentaria. Barcelona: Doyma S.A.

12. Castillo, R. (2011). Estomatología Pediátrica. Madrid, España: Ripano.

13. Castro, L., Galvao, A. (2004) Comparison of three different preparation methods in the improvement of selant retention. J Clin Pediatric Dent. 28(3):24952.

14. Cedillo, J. (2011). lonómeros de vidrio remineralizantes. Una alternativa de tratamiento preventivo o terapéutico. Revista ADM, 68 (5), 258265.

15. Chañ, D., Summit, J., García-Godoy, F. (1999) Evaluation of different methods for cleaning and preparing oclusal fissures. Oper Dent. 24(6):331-6.

16. Cova, J. (2010). Biomateriales dentales. Venezuela: Amolca.

17. Craig, R. (1998) Materiales de Odontología Restauradora. Edición 10. Editorial Harcour Brace. Madrid, Epaña.

18. Diéguez, E., Pascual, F., Vela, C. (2009). Selladores de fosas y fisuras para higienistas dentales y técnicas de colocación. España: Vigo.

19. Dominick, P. (1981). Odontología Preventiva. Editorial MundiSaic. Buenos Aires, Argentina.

20. Alave, G. (2014). Estudio in vitro: Evaluación con microscopio electrónico de barrido de la microfiltración marginal en un sellante de resina y uno de ionómero de vidrio modificado con resina, aplicado en fosas y fisuras de premolares en el periodo Setiembre - Noviembre (Tesis de pregrado). Recuperado de http://repositorio.unjbg.edu.pe/handle/UNJBG/2022

21. Utreras, V. (2016). Microfiltración de sellantes de fosas y fisuras: valoración de la microfiltración al microscopio de dos sellantes de diferente composición, sellante de resina y de lonómero de vidrio, en piezas definitivas premolares extraídas, estudio in vitro (Tesis de pregrado). Recuperado de http://www.dspace.uce.edu.ec/handle/25000/5749

22. Rojas (2018). Microfiltración en interfase dientesellante con y sin ameloplastía para dientes humanos con sistema autopolimerizable y fotopolimerizable. (Tesis de pregrado). Recuperado de http://repositorio.unfv.edu.pe/handle/UNFV/2590

\author{
Jéssica Avilés Erazo \\ Ana Armas Vega \\ Paola Mena Silva \\ Sebastián Chiluisa Cueva \\ Viviana Hidalgo Moya Isadora Martini García \\ Fabricio Mezzomo Collares \\ Edison López Ríos
}

